\title{
Optimum Bayesian Detection Based Interleaving Iteration Technology for Adjacent Cell Interference Mitigation
}

\author{
Ouyang Guojun \\ Department of Computer \\ Guangdong AIB Polytechnic College \\ Guangzhou, China \\ gzoygj@163.com
}

\author{
Qian Ye \\ School of Information Science and Technology \\ Fudan University \\ Shanghai
}

\begin{abstract}
In the communication of TD-LTE system, increase in frequency resource utilization to improve the system carrying capacity at the same time, resulting in the distance between the cells with the same frequency decreases with increasing from adjacent cells with the same channel interference. This article mainly aims at the fourth generation mobile communication system (TD - LTE) adjacent cell interference suppression and the OFDM - IDMA - OB (Optimum Bayesian Detection) multiuser joint Detection algorithm helps to improve the anti-interference ability of cell edge users. The performance improvement of the system is verified by using MATLAB to carry out bit error rate simulation.
\end{abstract}

Keywords-Adjacent cell interference,Optimal Bayesian Detection, IDMA,OFDM

\section{INTRODUCTION}

TD-LTE standard is the fourth generation mobile communication system with independent intellectual property rights in our country ${ }^{[1]}$. To August 2012, with the expansion of the scale of domestic TD-LTE to test the depth of the work, to further expand the scale of the TD-LTE test network, has been a good trial feedback. After several years of the concept of validation, basic testing and several commercial pilot test, the TD-LTE side of the commercial industrial chain has gradually improved.

As with other communication systems, TD-LTE is also unavoidable to face the problem of interference ${ }^{[2-3]}$. The OFDM (orthogonal frequency division multiplexing) used in TD-LTE can solve the problem of user's interference in the cell, but it brings the problem of cell interference. Especially for the cell edge users, between adjacent cell will exist a lot of overlapping bands, and far away from the base station location, the received signal to noise ratio is low, cell edge user of the received signal quality is bad. At present, the interference suppression of adjacent cells is a hot research topic at present, and also one of the key problems to be solved ${ }^{[4]}$. Many scholars are exploring new ways to find a solution to solve the problem. Can eliminate the interference of adjacent areas has become a key factor in the TD-LTE system can play a good performance.

In this paper, the design of the OFDM-IDMA based neighbor cell interference suppression transmitter and receiver structure is demonstrated and introduced, and for the downlink, a new detection algorithm based on the optimal Bias is proposed to eliminate the interference between cells, then the performance of the system is verified by using MATLAB to carry out the bit error rate simulation.

\section{SYSTEM MODEL ${ }^{[5-6]}$}

At the present stage of the OFDM-IDMA system, the uplink performance has been relatively complete, and can provide a good bit error rate and channel capacity. However, the interference suppression technology in the downlink is still less, which basically stays in the last generation mobile communication CDMA system. The system for TD-LTE system downlink use ofdm-idma technology, utilizing BPSK coding design a detection algorithm based on Bayesian optimal detection, the simulation results show that the receiver can improve the anti-interference ability of the cell edge user signal, the detection performance is good.

Orthogonal frequency division multiplexing (OFDM) can be a broadband channel is divided into number of positive subchannels, high-speed data signal is converted into parallel low-speed data flow, modulation in each sub channel for transmission. The transmission bandwidth is divided into a series of orthogonal sub carrier resources, and different sub carrier resources are allocated to different users to achieve multiple access. OFDM is capable of converting a wideband frequency selective channel into a set of parallel flat frequency sub carrier channels. Further, it is a signal by means of time domain equalization and not offset frequency selection channel $H_{I S I}$, circulating channel matrix $H_{C}$ is by over channels with memory has been transferred to the CP (cyclic prefix) is composed. The eigenvalue decomposition of the cyclic matrix channel $H_{C}$ is:

$$
H_{c}=U^{H} \Lambda U
$$

$\Lambda$ is the characteristic value of the diagonal matrix of $H_{C}, U$ is $H_{C}$ a single row vector. In fact feature vector $h_{k}$ $H$ is by in the transmitter and receiver respectively using fast Fourier transform (FFT) and inverse transform (IFFT) of the. The result is equivalent to the channel transfer function (FDCTF) in the frequency domain:

$$
G=F H F^{H}=\Lambda
$$


The signal $y_{k}$ of the $\mathrm{K}$ user is a character, which is generated by the $C_{k}$ of the user's channel code $r$ at rate $D_{k}=C_{k}\left(y_{k}\right)$. In IDMA system, because all users may use the same channel coding, so in order to distinguish the user defined $D_{k}=\pi_{k}\left[C\left(y_{k}\right)\right]$. In the IDMA environment, a feature length code is more or less experienced in the fast $\mathrm{Fu}$ Liye transform and inverse transform prior to each sub carrier channel. Generally speaking, the M subcarriers in orthogonal frequency divided multiplexing (OFDM) of the $\mathrm{j}$ a signal cycle of the $\mathrm{K}$ users of parallel orthogonal frequency division multiplexing standard discrete-time baseband channel model as shown in Figure 2-1, expressions are as follows:

$$
y[m, j]=\sum_{k=1}^{K} h_{k}[m, j] d_{k}[m, j]+n[m, j]
$$

Where $d k \in\{ \pm 1\}, y$ and $n \sim C N\left(0, N_{0}\right)$ represent the binary phase shift keying frequency domain signal, the received signal and additive white noise, whose variance is $\sigma_{n}^{2}=N_{0} / 2$. In addition, the frequency domain channel transfer function (FDCTF) of the user $\mathrm{K}$ is represented by $h_{k}$.

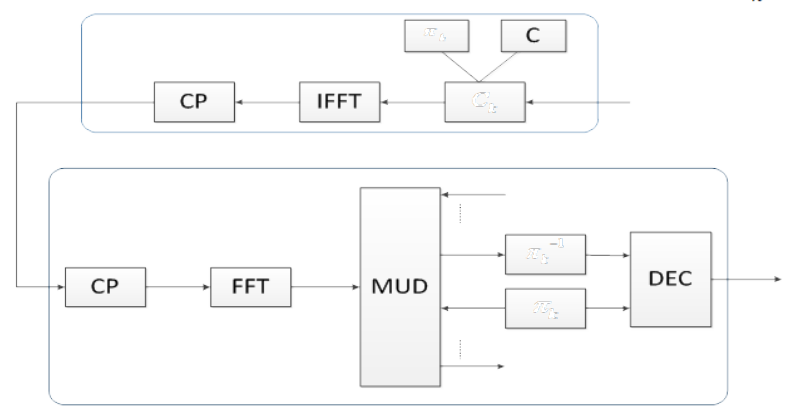

Figure 2-1 Transceiver model of the system

\section{OPTIMAL BAYESIAN ALGORITHM}

For the system, the frequency domain channel transfer function (FDCTs) is simulated by using a perfect iterative receiver. The long code chip is converted to the MUD and DEC two parts, see figure 3-1 ${ }^{[14]}$. Since then, focus on detection algorithm, considering the 1 symbol period of OFDM sub carrier, and then eliminate the sub carrier index $\mathrm{m}$ and OFDM symbol index j. Let $H=\left[h_{1}, \ldots, h_{k}\right], X=\left[x_{1}, \ldots x_{k}\right]^{\mathrm{T}}$. The subscript $(.)^{\mathrm{T}}$ denotes transpose.

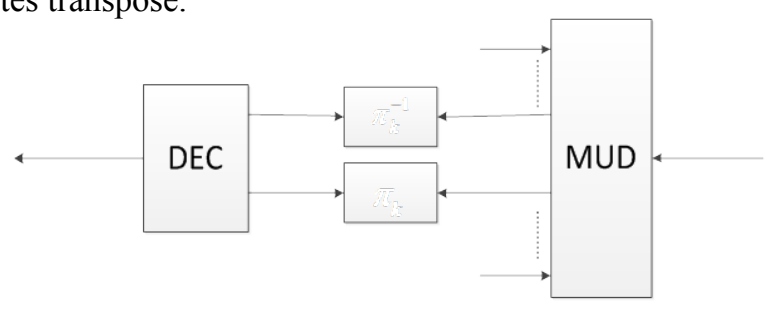

Figure 3-1 Receiver structure diagram
$L_{m u d}^{a}(x)$ is used to represent the log likelihood ratio (extrinsic information) of the prior information, and the external information is transmitted to the outer channel decoder via MUD $L_{m u d}^{e}(x)$.

Information based on each user is independent, so the log likelihood ratio of the external information of the $\mathrm{K}$ user is $L_{m u d}^{e}\left(x_{k}\right)=L_{m u d}\left(x_{k}\right)-L_{m u d}^{a}\left(x_{k}\right)$. Is the decision to receive signal detection. The log likelihood ratio is the decision threshold ${ }^{[7]}$.

The external information $L_{m u d}^{e}\left(x_{k}\right)$, which can be used to estimate the (3-1), is discussed. It can be estimated using formula (3-1).

$$
L_{\text {mud }}^{e}\left(x_{k}\right)=\ln \frac{p\left(y \mid x_{k}=+1, X_{-k}\right)}{p\left(y \mid x_{k}=-1, X_{-k}\right)}
$$

Which $X_{-k}$ said to rule out the k element in the $X$. The conditional probability density function $p\left(y \mid x_{k}= \pm 1, X_{-k}\right)$ can be obtained by calculating the $P\left(x_{k}= \pm 1\right)$ of the $p\left(y, x_{k}= \pm 1, X_{-k}\right)$ from the joint probability density function.

$$
\begin{aligned}
p\left(y \mid x_{k}= \pm 1, X_{-k}\right) & =\frac{p\left(y \mid x_{k}= \pm 1, X_{-k}\right)}{P\left(x_{k}= \pm 1\right)} \\
& =\sum_{X: x_{k}= \pm 1} \frac{p(y \mid X) P(X)}{P\left(x_{k}= \pm 1\right)} \\
& =\sum_{X: x_{k}= \pm 1} p(y \mid X) \prod_{i \neq k} P\left(x_{i}\right)
\end{aligned}
$$

When the information of each user is independent of the type set up. A priori probability $P\left(x_{i}\right)$ can be obtained from

the DEC of the MUD by means of the post interleaving feedback to the $L_{d e c}^{e}\left(x_{i}\right)$, Can be expressed as a formula (3-3).

$$
\begin{aligned}
P\left(x_{i}\right. & = \pm 1)=\frac{\exp \left[ \pm L_{d e c}^{e}\left(x_{i}\right)\right]}{1+\exp \left[ \pm L_{d e c}^{e}\left(x_{i}\right)\right]} \\
& =\frac{\exp \left[ \pm L_{d e c}^{e}\left(x_{i}\right) / 2\right]}{\exp \left[\mp L_{d e c}^{e}\left(x_{i}\right) / 2\right]+\exp \left[ \pm L_{d e c}^{e}\left(x_{i}\right) / 2\right]} \\
& =\cosh \left[L_{d e v}^{e}\left(x_{i}\right) / 2\right] \frac{1 \pm \tanh \left[L_{d e c}^{e}\left(x_{i}\right) / 2\right]}{2 \cosh \left[L_{d e c}^{e}\left(x_{i}\right) / 2\right]} \\
& =\frac{1}{2} \pm \frac{1}{2} \tanh \left[L_{d e c}^{e}\left(x_{i}\right) / 2\right]
\end{aligned}
$$

In addition, the conditional likelihood function $p(y \mid X)$ can be expressed as a formula (3-4). 


$$
p(y \mid X) \propto \exp \left(-\frac{\|y-H X\|^{2}}{2 \sigma_{n}^{2}}\right)
$$

$\propto$ is proportional to. Therefore, external information can be expressed as (3-5).

$$
\begin{aligned}
L_{\text {mud }}^{e}\left(x_{k}\right) & =\ln \frac{p\left(y \mid x_{k}=+1, X_{-k}\right)}{p\left(y \mid x_{k}=-1, X_{-k}\right)} \\
& =\ln \frac{\sum_{X: x_{k}=+1} \exp \left(-\|y-H X\|^{2} / 2 \sigma_{n}^{2}\right) \varphi}{\sum_{X: x_{k}=-1} \exp \left(-\|y-H X\|^{2} / 2 \sigma_{n}^{2}\right) \varphi}
\end{aligned}
$$

Among them, $\varphi=\prod_{i \neq k} P\left(x_{i}\right), \quad P\left(x_{i}\right)$ by (3-4) type is given. Then the information $L_{\text {mud }}^{e}\left(x_{k}\right)$ is sent to the DEC by the solution ${ }^{[8]}$.

\section{SIMULATION RESULTS AND ANALYSIS}

The performance of the system is compared with that of the Gauss white noise channel by using the OFDM-IDMA receiver with the BPSK code. First consider the transmit power of each base station is the same, the fading channel is inversely proportional to the square of the distance, when the user in the cell edge to consider their acceptance of different signal to noise ratio of the signal generated by error simulation. In which the ratio of the information energy and all the noise energy is received by all the receivers except the coded information, the ratio of all the noise energy is used as the parameters of the system performance.

In this paper, the system performance is simulated in the Gauss white noise (AWGN) channel, which is suppressed by the neighbor cell interference suppression of 4 users when using the spreading factor of 4 . Results are as shown in figure 4-1.

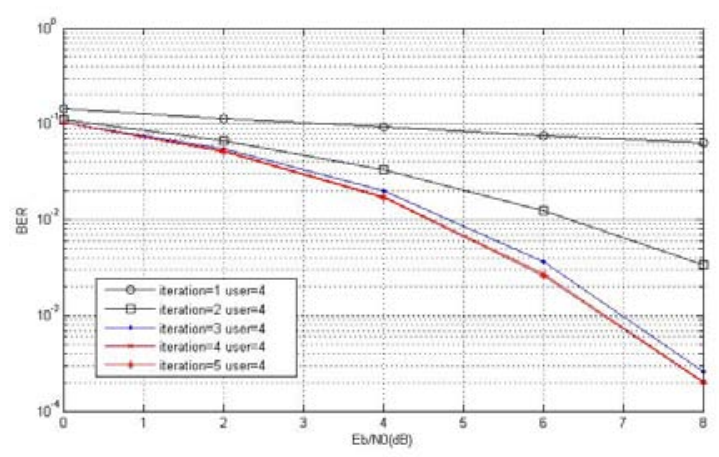

Figure 4-1 Simulation results of different iteration times under the condition of 4 spread spectrum coefficient

Can be found from Figure 4-1, with the increase of the number of iterations. The error rate of the system is rapidly reduced, the iterative receiver is fast convergence, when the number of iterations to reach 4 times the system performance tends to be stable, the system performance is no longer.

At this point, the system will achieve the desired results only two times. By observing the first iteration of the 3 systems when the signal to noise ratio is $6 \mathrm{~dB}$, we can know that, with the increase of the spread spectrum code, the iteration has brought about a faster performance improvement. Results are as shown in figure 4-2.

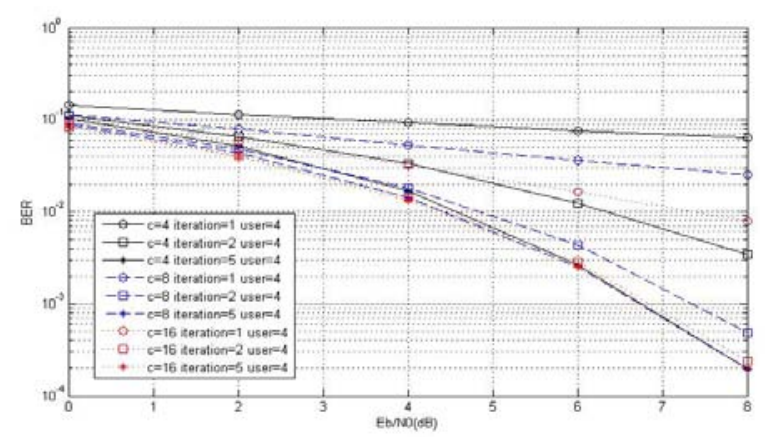

Figure 4-2 Simulation results of different iteration times under the condition of 4816 spread spectrum coefficient

From Figure 4-2, it can be seen that the 1 iterations and 2 iterations have obvious changes in different spread spectrum codes, and the convergence of the spread code is more obvious. However, when the number of iterations increased to 5 times and more, the basic convergence in a line, spread spectrum code no longer make the performance of a greater increase.

Spread spectrum code contains redundant information, when the spread spectrum code increases, information in the feedback will contain more redundant information, it is the redundant information and enhance the system in every iteration to acquire faster performance.

\section{CONCLUSION}

Is introduced in this paper adjacent cell interference suppression system model, and according to the downlink, using an iterative receiver, using the optimal Bayesian algorithm to design the new estimation algorithm, finally, on the basis of the algorithm were the bit error rate (BER) of the Monte Carlo simulation, and analyzes results. From the results, it can be seen that the algorithm has a good effect on the adjacent cell interference, and the signal interference problem of the cell edge users can be well solved.

\section{Acknowledgment}

This paper is supported by the national science and technology major project (No.2012ZX03001013-004). 


\section{References}

[1] Shen Xingpeng,Tang Shoulian. The Analysis of TD-LTE Industry Value Chains in Chinese Mobile Communication Market[J]. IEEE Trans,2010: 166-169

[2] Xiaolin Li, Wenwen Liu. Downlink MIMO signal detection algorithm in TD-LTE system[J].Second International Conference on Business Computing and Global Informatization.,2012: 649-652.

[3] Deepak Pareek. The Business of WiMAX[M].England:John Wiley\&Sons, LTD, 2006.

[4] Weihong Fu,Chunbao Zhao,Wu Wei,Qingliang Kong. Improved Sphere Decoding Algorithm in TD-LTE System[C]. IEEE Trans, 2010: 514-517.
[5] M.M.Kamruzzaman. Performance of Turbo coded wireless link for SISO-OFDM, SIMO-OFDM, MISO-OFDM and MIMO-OFDM[C]. IEEE Trans,2011.

[6] Lei Haipeng, Zhang Lei, Zhang Xin, Yang Dacheng. A Novel MultiCell OFDMA System Structure Using Fractional Frequency Reuse[J]. PIMRC,2007

[7] Lei Xu, Rong Zhang, Sheng Chen,Lajos Hanzo. EXIT-Chart Aided Hybrid Multiuser Detector Design for Frequency-Domain-Spread ChipInterleaved MC-CDMA[J].IEEE Trans.2010:1816-1820.

[8] Rong Zhang. EXIT-Chart-Aided Hybrid Multiuser Detector for Multicarrier Interleave-Division Multiple Access[J].IEEE Trans.2010. 